cations," IEEE Trans. Commun., vol. COMM-27, pp. 224-236, Feb. 1987.

[21] M. Schwartz, W. Bennett, and S. Stein, Communication Systems and Techniques. New York: McGraw-Hill, 1966.

[22] S. Wang and I. Wang, "Effects of soft handoff, frequency reuse and non-ideal antenna sectorization on CDMA system capacity," in Proc. VTC '93, pp. 850-854.

[23] A. Jalali and P. Mermelstein, "Power control and diversity for the downlink of CDMA systems," in Proc. ICUPC '93, pp. 980-984.

\section{Distributed Power Balancing in Cellular Systems Using Limited Control-Data Flow}

\author{
Kam Hung Lam and Wing Shing Wong
}

\begin{abstract}
In this paper, a new, distributed power-control algorithm is introduced. The algorithm is based on the assumption that some limited control-data communication is allowed according to a predefined dataflow structure. The convergence property of this algorithm is analyzed. Numerical study results are also presented.
\end{abstract}

Index Terms - Cooperative algorithm, distributed algorithm, positive matrix, power control.

\section{INTRODUCTION}

Power control is an important issue in mobile communications that receives a lot of attention (for example, see [1]-[5]). The basic question is how to adjust the power level at each transmitter so that the carrier-to-interference $(\mathrm{C} / \mathrm{I})$ ratio is optimized in some global sense.

The power-control problem is complicated by the effect of fast fading, and it is common practice to divide this complicated problem into two parts. First, one tries to obtain a globally optimal solution ignoring the effect of fast fading. One can then design a close-loop control for the transmitter to combat the effect of fast fading. In this paper, we concentrate only on the first problem.

Since the C/I ratio can be defined at each receiver, there are a variety of ways to combine these values to form a single, global quality measure. One of the commonly used criterions is C/I balancing introduced by Aein [1]. Under this criterion, a system is optimally controlled if all the uplink (or downlink) channels sharing the same spectrum have identical C/I ratios. A balanced solution is also known to be the optimal solution to the problem of maximizing the minimum of all the individual C/I's.

Given the nature of the power-control problem, that involves transceivers that are geographically distributed, the solution should ideally be computable by each transceiver in isolation without having to communicate with other transceivers (the communication between a mobile unit and the base station to which it belongs is allowed, however). Unfortunately, most of the commonly known approaches to power control, such as those stated in [3]-[5], are not completely isolated algorithms. For example, the algorithms presented in [3] and [4] allow each transceiver to compute its power level in isolation. However, the algorithms do not converge unless a common normal-

Manuscript received August 12, 1994; revised July 1, 1995 and September 13, 1995.

The authors are with the Department of Information Engineering, Chinese University of Hong Kong, Shatin, NT Hong Kong.

Publisher Item Identifier S 0018-9545(97)00653-1. izing factor is chosen correctly. Hence, for these algorithms some global communication seems unavoidable in order to guarantee that the normalizing factors are chosen correctly.

In this paper, we introduce a new distributed power-control algorithm. Our algorithm is based on the assumption that some limited control-data communication between the interfering transceivers is allowed. The algorithm is distributed in the sense that it is computed by each transceiver based on its local information and information sent by some of its neighbors. Of course, such information exchanges generated some overhead. However, the trade-off is that the new algorithm has the following nice features.

1) It does not require a common normalizing factor that needs to be broadcast globally to all the mobile units.

2) It has the convergence property that the minimum of the $\mathrm{C} / \mathrm{I}$ ratio of all the interfering transceivers converges monotonically upward to a limit.

3) While its rate of convergence is comparable to the algorithm proposed in [4], fewer power adjustments are required. Moreover, it guarantees that there is no oscillation of power levels during the adjustment.

4) Power level and C/I ratio constraints are incorporated.

After the basic introduction, we introduce the concept of controldata flow structure that is essential in describing the intercell communication needed in the distributed algorithm. In Sections IV and V, the algorithm is presented and analyzed. The findings of our numerical study are discussed in Section VI.

In this paper, the effect of thermal noises is not modeled explicitly. This assumption simplifies the mathematics significantly and enables us a more lucid presentation of the key ideas.

\section{System MODEL}

Our model is based on the model presented in [1] that is briefly reviewed here for completeness. We study the scenario, where the mobile cellular communication system has $K$ base stations, each of which has one active user. For each user, there is a channel pair consisting of an uplink and a downlink channel. The mode of medium-access control on these channels can be frequency division multiple access (FDMA) or time division multiple access (TDMA). Neighboring channel interference is ignored.

Propagation loss and interference effects are captured by the link gains. The notation $G_{i j}$ is used to represent the path gain received at the base station $i$ from the transmitter of the mobile unit $i$. The matrix $G=\left\{G_{i j}\right\}$ is known as the uplink-gain matrix. One can define the downlink-gain matrix similarly. Since the power-control issue for the uplink and downlink channels are quite similar in our model, we concentrate only on the uplink channel power control to simplify the discussion. Note that $G_{i i}$ represents the path gain for the intended signal, while for $i \neq j, G_{i j}$ represents the path gain for the interference signals. Thus, the $\mathrm{C} / \mathrm{I}$ ratio received by base station $i$ and $\Gamma_{i}$ can be written as

$$
\Gamma_{i}=\frac{G_{i i} P_{i}}{\sum_{j \neq i} G_{i j} P_{j}} .
$$

We assume that this $\mathrm{C} / \mathrm{I}$ ratio reflects the transmission quality at the receiver; interference due to thermal noises is assumed to be negligible. Equation (1) can be rewritten more compactly as

$$
\Gamma_{i}=\frac{P_{i}}{\sum_{j \neq i} Z_{i j} P_{j}}
$$




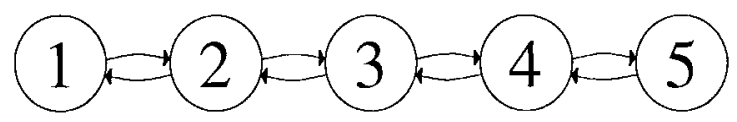

Fig. 1. An example of a control-data flow structure.

where the normalized uplink-gain matrix $Z$ is defined by $Z_{i j}=$ $G_{i j} / G_{i i}$.

As in [1], a C/I ratio $\gamma$ is defined to be achievable if power vector $\mathbf{P}$ exists with all positive components such that $\Gamma_{i} \geq \gamma$ for all $i$.

A matrix $A$ is called irreducible if there does not exist a permutation of the base that can put it in the form

$$
\left(\begin{array}{ll}
B & 0 \\
C & D
\end{array}\right)
$$

where $B$ and $D$ are square matrices. Since the normalized linkgain matrix is unlikely to contain any zero entry, we can assume without losing much generality that $Z$ is irreducible. By the Frobenius Theorem, $Z$ has a unique, dominant real eigenvalue $\lambda^{*}$. In [1], an important result is proved stating that the largest achievable $\mathrm{C} / \mathrm{I}$ ratio $\gamma^{*}$ is related to $\lambda^{*}$ by the formula

$$
\gamma^{*}=\frac{1}{\lambda^{*}-1} \text {. }
$$

Moreover, an eigenvector corresponding to the eigenvalue $\lambda^{*}$ yields a balanced solution of the power-control problem. This result reduces the problem of finding an optimal power-control solution to finding the corresponding eigenvector of the normalized gain matrix.

\section{Control-Data Flow Structure}

A crucial assumption in our model is that the base stations in the system are interconnected by a packet-switched signaling network so that a small amount of control-data information can be sent from one base station to another. Of course, there is a cost associated with this type of data communication, and, naturally, one wants to minimize the cost by ensuring that this type of data communication is kept to a minimum. Hence, the algorithm we propose is designed to restrict control-data traffic to network neighbors as much as possible. By network neighbors, we refer to those base stations between which the data communication costs are small.

A key concept in our distributed algorithm is the control-data flow structure. This structure can be represented as a directed graph. It defines how the power-control data are passed among the base stations. So, if there is a directed arc from node A to B, then control data is expected to be passed from base station A to B. The control-data flow structure is dictated by the topology of the signaling network, but otherwise it can be quite general except that it must satisfy the following.

\section{A. Reachability Condition}

A control-data flow structure satisfies the reachability condition if, for any pair of nodes $(A, B)$, there is a chain of directed arcs starting from $A$ and terminating at $B$.

An example of a control-data flow structure satisfying the reachability condition is provided in Fig. 1. Since we focus on the uplink channels in this paper, the $\mathrm{C} / \mathrm{I}$ ratios are assumed to be measured locally at the base stations. These C/I ratio measurements are averaged over a suitable time interval to smooth out fluctuations due to fast fading. At the beginning of each algorithm iteration, each base station passes the averaged $\mathrm{C} / \mathrm{I}$ ratio measurement to some of its neighbors according to the predefined control-data flow structure. This information is then used in the computation of the next iteration.

\section{The Distributed Power-Control Algorithm}

In this section, we define a simple distributed power-control algorithm. Before we proceed, let us recall some basic notation, most of which is borrowed from [1].

$B_{i} \quad i$ th base station; the user homing onto $B_{i}$ is labeled as the $i$ th user;

$K \quad$ total number of active base stations;

$\mathcal{K} \quad$ set of integers from 1 to $K$;

$\gamma_{0} \quad$ minimum $\mathrm{C} / \mathrm{I}$ ratio for transmission with acceptable quality;

$\Gamma_{i}^{(n)} \quad \mathrm{C} / \mathrm{I}$ ratio at the $i$ th base station at the $n$th iteration;

$P_{i}^{(n)} \quad$ transmitter power level of mobile unit $i$ at the $n$th iteration;

$P_{M} \quad$ maximum transmission power level of the mobile units;

$\mathcal{N}_{i} \quad$ set of indexes of base stations that send control-data information to base station $i$ according to the control-data flow structure;

$\overline{\mathcal{N}}_{i} \quad$ union of $\mathcal{N}_{i}$ with $i$.

The distributed power-control algorithm we propose is a discretetime algorithm. At each iteration, every base station computes its own power level based on its power level in the previous iteration, its current $\mathrm{C} / \mathrm{I}$ ratio, and the $\mathrm{C} / \mathrm{I}$ ratios it receives from its neighbors. Hence, it is assumed that the computations are synchronized. The issue of asynchronous updates, such as in [7], is not considered in this paper. To be more precise, the new algorithm is defined by the following set of equations.

\section{A. The Cooperative Algorithm}

Each mobile unit adjusts its power according to the following rules:

$$
\begin{gathered}
P_{i}^{(0)}=P_{M} \\
P_{i}^{(n+1)}=\alpha_{i}^{(n)} P_{i}^{(n)}
\end{gathered}
$$

where $\alpha_{i}^{(n)}$ is defined to be

$$
\alpha_{i}^{(n)}=\sqrt[m]{\frac{\min \left(\Gamma_{i}^{(n)}, \max \left(\min _{j \in \mathcal{N}_{i}} \Gamma_{j}^{(n)}, \gamma_{0}\right)\right)}{\Gamma_{i}^{(n)}}}
$$

and $m$ is a parameter of the algorithm that controls the rate of convergence as explained in the numerical study section.

The use of the $m$ th square root function in the definition of $\alpha_{i}^{(n)}$ is motivated by the fact that for a system with only two base stations, it is possible to reach the balanced state in one iteration by choosing $\alpha_{i}^{(n)}$ as a function of the form

$$
c \sqrt{\frac{\min _{j=1,2} \Gamma_{j}^{(n)}}{\Gamma_{i}^{(n)}}} .
$$

A detailed discussion of this result is given in the Appendix.

\section{Convergence Properties of the Cooperative Algorithm}

We summarize the convergence properties of the cooperative algorithm in a series of propositions and a theorem.

Proposition 1: The power level $P_{i}^{(n)}$ is a monotone nonincreasing function of $n$. Moreover, the function

$$
\gamma(n)=\min _{j \in \mathcal{K}} \Gamma_{j}^{(n)}
$$

is a monotone nondecreasing function of $n$. 
Proof: A function $f$ is monotone nondecreasing if $f(x) \leq f(y)$ whenever $x \leq y$. A monotone nonincreasing function is defined similarly. The fact that $P_{i}^{(n)}$ is monotone nonincreasing follows simply from the fact that $\alpha_{i}^{(n)} \leq 1$.

To show the second statement, note that

$$
\begin{aligned}
\Gamma_{i}^{(n+1)} & =\frac{\alpha_{i}^{(n)} P_{i}^{(n)}}{\sum_{j \neq i} Z_{i j} P_{j}^{(n+1)}} \geq \frac{\alpha_{i}^{(n)} P_{i}^{(n)}}{\sum_{j \neq i} Z_{i j} P_{j}^{(n)}} \\
& =\alpha_{i}^{(n)} \Gamma_{i}^{(n)}=\sqrt[m]{\beta_{i}^{(n)}\left(\Gamma_{i}^{(n)}\right)^{m-1}}
\end{aligned}
$$

where

$$
\beta_{i}^{(n)}=\min \left(\Gamma_{i}^{(n)}, \max \left(\min _{j \in \mathcal{N}_{i}} \Gamma_{j}^{(n)}, \gamma_{0}\right)\right) .
$$

It follows from (8) that

$$
\Gamma_{i}^{(n+1)} \geq \min \left(\Gamma_{i}^{(n)}, \max \left(\min _{j \in \mathcal{N}_{i}} \Gamma_{j}^{(n)}, \gamma_{0}\right)\right) .
$$

If $\min _{j \in \mathcal{N}_{i}} \Gamma_{j}^{(n)} \geq \gamma_{0}$, then

$$
\Gamma_{i}^{(n+1)} \geq \min \left(\Gamma_{i}^{(n)}, \min _{j \in \mathcal{N}_{i}} \Gamma_{j}^{(n)}\right)=\min _{j \in \mathcal{N}_{i}} \Gamma_{j}^{(n)} .
$$

On the other hand, if $\min _{j \in \mathcal{N}_{i}} \Gamma_{j}^{(n)}<\gamma_{0}$, then

$$
\Gamma_{i}^{(n+1)} \geq \min \left(\Gamma_{i}^{(n)}, \gamma_{0}\right) \geq \min _{j \in \overline{\mathcal{N}}_{i}} \Gamma_{j}^{(n)}
$$

Hence, in either case, $\gamma(n+1) \geq \gamma(n)$.

Since $P_{i}^{(n)}$ is a monotone nonincreasing sequence with zero as a lower bound, it follows that the limit of $P_{i}^{(n)}$ exists as $n$ tends to infinity. Denote the limit by $P_{i}^{*}$. Denote the corresponding value of the $\mathrm{C} / \mathrm{I}$ ratio at the $i$ th base station as $\Gamma_{i}^{*}$. Denote the limit of $\alpha_{i}^{(n)}$ accordingly as $\alpha_{i}^{*}$. Although we have shown that the limiting power control exists, in general, we cannot establish the fact that the limit does not tend to zero. In our numerical studies, however, the power limits we encountered have always been nonzero. In the rest of this paper, we will always assume that the limiting power levels are all positive.

Recall that $\gamma^{*}$ is the dominant real eigenvalue of the normalized uplink-gain matrix $Z$. We have the following result.

Proposition 2: If $\Gamma_{i}^{*} \geq \gamma_{0}$ for all $i$, then $\Gamma_{i}^{*}=\gamma^{*}$, and the balanced solution is obtained.

Proof: Since $\Gamma_{i}^{*}$ is the limiting C/I ratio, it follows that

$$
\Gamma_{i}^{*}=\frac{\alpha_{i}^{*} P_{i}^{*}}{\sum_{j \neq i} Z_{i j} P_{j}^{*}}=\alpha_{i}^{*} \Gamma_{i}^{*} .
$$

Hence, $\alpha_{i}^{*}=1$. This, in turn, implies that for any $i$

$$
\Gamma_{i}^{*}=\min \left(\Gamma_{i}^{*}, \max \left(\min _{j \in \mathcal{N}_{i}^{*}} \Gamma_{j}^{*}, \gamma_{0}\right)\right)=\min _{j \in \mathcal{\mathcal { N }}_{i}} \Gamma_{j}^{*} .
$$

Let $B_{k}$ be a base station that receives the globally maximal $\mathrm{C} / \mathrm{I}$ ratio. By the reachability assumption, for any node $i, i \neq k$, there is a chain of directed arcs starting from node $k$ and ending at node $i$. Label the nodes in the chain as $j_{0}=k, j_{1}, j_{2}, \cdots, j_{l}=i$. Since $j_{1} \in \mathcal{N}_{k}$, it follows that

$$
\Gamma_{k}^{*} \leq \Gamma_{j_{1}}^{*} .
$$

Similarly, one can show that

$$
\Gamma_{k}^{*} \leq \Gamma_{j_{1}}^{*} \leq \Gamma_{j_{2}}^{*} \cdots \leq \Gamma_{i}^{*}
$$

Since $\Gamma_{k}^{*}$ achieves the globally maximal value, the strict equality holds in the previous inequalities and $\Gamma_{i}^{*}=\Gamma_{k}^{*}$ for all $i$.
Proposition 3: If there exists a base station $k$ such that $\Gamma_{k}^{*}<\gamma_{0}$, then

1) $\Gamma_{i}^{*} \leq \gamma_{0}$ for all $i$

2) $\Gamma_{i}^{*}=\gamma_{0}$ for any $B_{i}$ satisfying $\Gamma_{i}^{(0)} \geq \gamma_{0}$.

Proof: As before, it follows from (13) that $\alpha_{i}^{*}=1$ for any base station $i$. If $B_{i}$ has a neighbor $B_{j}$ such that $\Gamma_{j}^{*} \leq \gamma_{0}$, then $\alpha_{i}^{*}=1$ implies

$$
\Gamma_{i}^{*}=\min \left(\Gamma_{i}^{*}, \gamma_{0}\right)
$$

Hence, $\Gamma_{i}^{*} \leq \gamma_{0}$. By the reachability assumption, for any node $i, i \neq k$, there is a chain of directed arcs starting from node $k$ and ending at node $i$. Thus, by inductively applying the previous argument on the nodes along this chain, one can establish the first statement.

It follows from the definition of $\beta_{i}^{(n)}$ that if $\Gamma_{i}^{(n)} \geq \gamma_{0}$, then $\beta_{i}^{(n)} \geq \gamma_{0}$. Thus, (8) implies that $\Gamma_{i}^{(n+1)} \geq \gamma_{0}$. So, if $\Gamma_{i}^{(0)} \geq \gamma_{0}$, then $\Gamma_{i}^{(n)} \geq \gamma_{0}$ for any positive integer $n$. Hence, $\Gamma_{i}^{*} \geq \gamma_{0}$. Combining this result with the first statement, one concludes that

$$
\Gamma_{i}^{*}=\gamma_{0}
$$

if $\Gamma_{i}^{(0)} \geq \gamma_{0}$.

Theorem: Assume that the limiting power-control vector is nonzero. If $\gamma^{*} \geq \gamma_{0}$, then the cooperative algorithm converges to the balanced solution that is $\Gamma_{i}^{*}=\gamma^{*}$ for all base stations. If $\gamma^{*}<\gamma_{0}$, then $\Gamma_{i}^{*} \leq \gamma_{0}$ for all base stations, with the strict inequality holding for at least one base station. Moreover, if $\Gamma_{i}^{(0)} \geq \gamma_{0}$, then $\Gamma_{i}^{*}=\gamma_{0}$.

Proof: To establish the theorem, let us first show that if $\Gamma_{i}^{*} \geq \gamma_{0}$ for all $i$, then $\gamma^{*} \geq \gamma_{0}$, and if $\Gamma_{k}^{*}<\gamma_{0}$ for some $k$, then $\gamma^{*}<\gamma_{0}$. If this holds, then the condition $\gamma^{*} \geq \gamma_{0}$ implies $\Gamma_{i}^{*} \geq \gamma_{0}$ for all $i$, and $\gamma^{*}<\gamma_{0}$ implies $\Gamma_{k}^{*}<\gamma_{0}$ for some $k$. The theorem then follows from Propositions 2 and 3.

By a corollary of the Frobenius Theorem [6], it follows that for any power-control assignment with all positive components $\mathbf{P}$, the $\mathrm{C} / \mathrm{I}$ ratios defined by

$$
\Gamma_{i}(\mathbf{P})=\frac{\mathbf{P}_{i}}{\sum_{j \neq i} Z_{i j} \mathbf{P}_{j}}
$$

satisfy the condition

$$
\min _{i \in \mathcal{K}} \Gamma_{i}(\mathbf{P}) \leq \gamma^{*} \leq \max _{i \in \mathcal{K}} \Gamma_{i}(\mathbf{P})
$$

It follows in particular that

$$
\min _{i \in \mathcal{K}} \Gamma_{i}^{*} \leq \gamma^{*} \leq \max _{i \in \mathcal{K}} \Gamma_{i}^{*}
$$

As a corollary of the Frobenius Theorem, if either one of the inequalities in (18) can be replaced by an equality, then $\mathbf{P}$ is an eigenvector of $\gamma^{*}$ and $\min _{i \in \mathcal{K}} \Gamma_{i}^{*}=\gamma^{*}=\max _{i \in \mathcal{K}} \Gamma_{i}^{*}$.

If $\Gamma_{k}^{*}<\gamma_{0}$ for some $k$, then

$$
\min _{i \in \mathcal{K}} \Gamma_{i}^{*}<\gamma_{0}
$$

It follows from Proposition 3 that for all $i$

$$
\Gamma_{i}^{*} \leq \gamma_{0}
$$

Equation (20) implies that $\gamma^{*} \leq \gamma_{0}$. If $\gamma^{*}=\gamma_{0}$, then $\gamma^{*}=$ $\max _{i \in \mathcal{K}} \Gamma_{i}^{*}$ and $\min _{i \in \mathcal{K}} \Gamma_{i}^{*}=\max _{i \in \mathcal{K}} \Gamma_{i}^{*}$ : a contradiction to (19). Hence, $\gamma^{*}<\gamma_{0}$.

On the other hand, if $\Gamma_{i}^{*} \geq \gamma_{0}$ for all $i$, then by Proposition 2, the balanced solution is obtained. So

$$
\gamma^{*}=\Gamma_{i}^{*} \geq \gamma_{0}
$$


TABLE I

Average Number of Iterations to Achieve the Specified Target Averaged Over 1000 Experiments. Mean Value of Balanced C/I $=19.1 \mathrm{~dB}$

\begin{tabular}{|c|c|c|c|}
\hline & within $92 \%$ & within 95\% & within 98\% \\
\hline Cooperative Algorithm, structure $S_{1}, m=2$ & 87.83 & 99.51 & 120.90 \\
\hline Cooperative Algorithm, structure $S_{2}, m=2$ & 8.19 & 10.35 & 15.04 \\
\hline Cooperative Algorithm, structure $S_{2}, m=1.1$ & 9.52 & 11.32 & 14.98 \\
\hline Algorithm in [4] & 21.09 & 24.17 & 30.02 \\
\hline
\end{tabular}

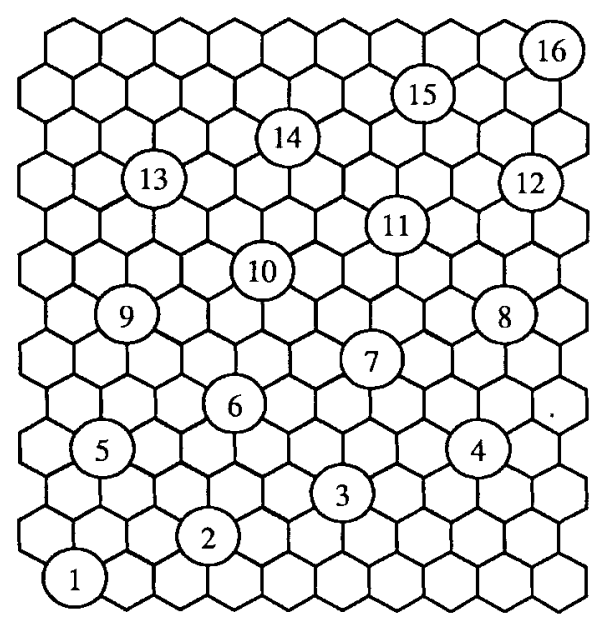

Fig. 2. Layout of interfering cells. In the numerical study, interfering cells are assumed to be circular.

\section{NUMERICAL STUDY}

We have carried out a numerical study on the cooperative algorithm. Our simulation model is similar to the model in [3]. The standard hexagonal cell layout is assumed. The radius of the cells is normalized to one. A fixed, homogeneous channel-allocation strategy is used with a reuse factor of $N=7$. The distance between two neighboring cells, $D$, is determined by the well-known formula

$$
D=R \sqrt{3 N}
$$

where $R$ is the cell radius. Since $R$ is normalized to be one, $D=\sqrt{21}$. The base stations are assumed to be located at the center of the cells. Users in the 16 circular cells marked in Fig. 2 are assumed to use the same bandwidth, and power control is required to minimize the cochannel interference among them.

To simulate the users, the location of a mobile unit is generated randomly by picking a set of polar coordinates $(r, \theta)$ so that $r$ is uniformly chosen on the interval $[0,1]$ and $\theta$ is uniformly chosen from $[0,2 \pi]$. The polar coordinate is then used to define the relative position of the $i$ th mobile unit from the center of the $i$ th cell. The link-gain value $G_{i j}$ is then defined by the formula

$$
G_{i j}=\frac{A_{i j}}{d_{i j}^{4}}
$$

where $d_{i j}$ is the distance between the $i$ th base station and the $j$ th mobile unit and $A_{i j}$ is the attenuation factor.

As we mentioned before, we only consider the effect of log-normal fading and ignore the effect of fast fading in this study. Hence, for all $i$ and $j, A_{i j}$ is log-normal distributed with

$$
\mathbf{E}\left[10 \log _{10} A_{i j}\right]=0 d B
$$

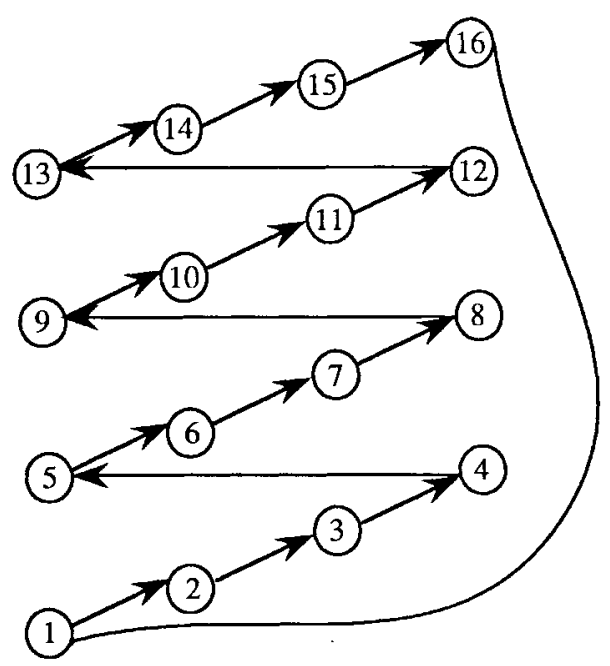

(a)

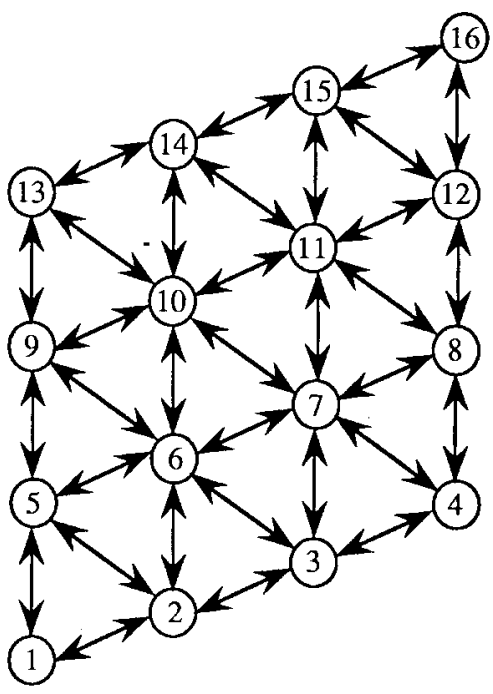

(b)

Fig. 3. Data-flow control structure studied in this paper.

$$
\operatorname{Var}\left[10 \log _{10} A_{i j}\right]=\sigma^{2}=(6 d B)^{2} .
$$

Two types of control-data flow structure were considered. Structure $S_{1}$ is represented by a directed tour where node 1 is joined to node 2 , node 2 is joined to node 3 , and so on (node 16 is joined to node 1 ). In structure $S_{2}$, every cell sends its $\mathrm{C} / \mathrm{I}$ information to its geographical neighbors as shown in Fig. 3. While $S_{1}$ is one of the minimal structures possible that satisfies the reachability condition, 


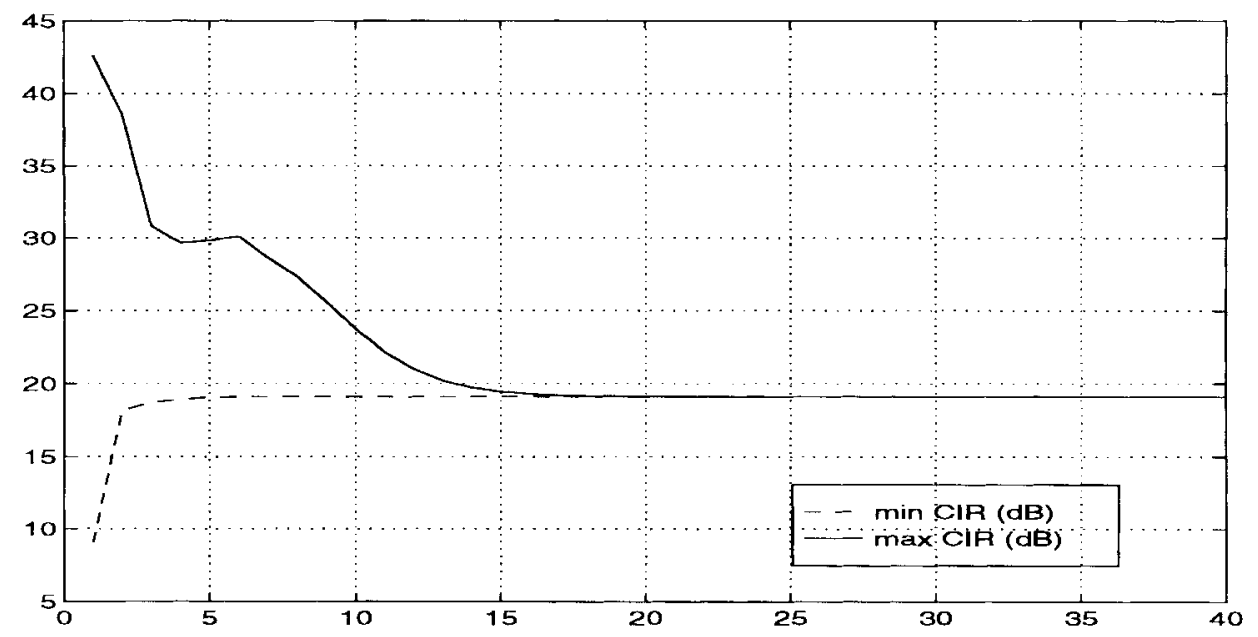

Fig. 4. Rate of convergence of the cooperative algorithm. Minimum and maximum C/I ratios of cells using the cooperative algorithm with the $S_{2}$ structure are displayed.

TABLE II

Average Maximum and Minimum Power Levels to Achieve the Specified Target Averaged Over 1000 Experiments. Mean Value of Balanced C/I $=19.1 \mathrm{~dB}$

\begin{tabular}{|c|c|c|c|}
\hline & within $92 \%$ & within $95 \%$ & within $98 \%$ \\
\hline Cooperative Algorithm, structure $S_{1}, m=2$ & $0.37,2.2 \mathrm{e}-4$ & $0.35,1.8 \mathrm{e}-4$ & $0.33,1.6 \mathrm{e}-4$ \\
\hline Cooperative Algorithm, structure $S_{2}, m=2$ & $0.79,1.2 \mathrm{e}-3$ & $0.77,9.0 \mathrm{e}-4$ & $0.75,5.8 \mathrm{e}-4$ \\
\hline Cooperative Algorithm, structure $S_{2}, m=1.1$ & $0.17,1.2 \mathrm{e}-4$ & $0.16,9.0 \mathrm{e}-5$ & $0.15,6.5 \mathrm{e}-5$ \\
\hline Algorithm in $[4]$ & $2.2 \mathrm{e}-4,1.5 \mathrm{e}-7$ & $9.4 \mathrm{e}-5,3.1 \mathrm{e}-8$ & $3.2 \mathrm{e}-7,2.6 \mathrm{e}-11$ \\
\hline
\end{tabular}

its corresponding algorithm has a rate of convergence about an order of magnitude slower than one that uses $S_{2}$. Hence, $S_{1}$ is deemed unacceptable.

A large number of simulation runs were conducted. In Figs. 4 and 5, we present some typical results seen in these experiments. These figures compare the cooperative algorithm with the distributed algorithm proposed in [4] that is known to have a fast rate of convergence. The value of $m$ was chosen to be 1.5 , and $\gamma_{0}$ was set to zero. In Fig. 4, the rate of convergence of the new algorithm using data structure $S_{2}$ is presented. In Fig. 5, the rate of convergence using the algorithm in [4] is presented. The different rates of convergence for the different algorithms are also summarized in Table I, based on 1000 simulation runs.

In Table II, we compare the average minimum and maximum power levels derived by the algorithms when the $\mathrm{C} / \mathrm{I}$ ratio is within target. Since the dynamic range of the power level is more important that its absolute value, we normalize the maximum power in both algorithms to one; in particular, this means $P_{M}$ is equal to one. The study shows that for the algorithm proposed in [4], the final power levels are almost zero. So, in practice, the algorithm must terminate before the equilibrium can be reached. For our algorithm, the derived power levels are more realistic. Again, structure $S_{2}$ provides better performance than $S_{1}$. Moreover, the rate of convergence for $S_{2}$ is faster than the rate of convergence of the algorithm in [4], while the rate of convergence for $S_{1}$ is slower than both.

In Table III, the effects of different $m$ values are summarized. The configuration of the system is identical to previous experiments. The results indicate that the optimal value for $m$ to minimize the number of iterations is around 1.5. This is slightly different from the theoretical optimum value for a system consisting of two cells,
TABLE III

A Comparison of the EFFect of $m$ on the Convergence Rate

\begin{tabular}{|c|c|c|c|}
\hline$m$ value & $\begin{array}{c}\text { Avg. \# of iterations to } \\
98 \% \text { of balanced value }\end{array}$ & $\begin{array}{c}\text { Mean maximum } \\
\text { power level }\end{array}$ & $\begin{array}{c}\text { Mean minimum } \\
\text { power level }\end{array}$ \\
\hline 1.1 & 14.98 & 0.15 & $6.5 \mathrm{e}-5$ \\
\hline 1.5 & 11.94 & 0.57 & $3.8 \mathrm{e}-4$ \\
\hline 2 & 15.04 & 0.75 & $5.8 \mathrm{e}-4$ \\
\hline 4 & 30.79 & 0.83 & $5.3 \mathrm{e}-4$ \\
\hline 8 & 63.39 & 0.90 & $7.7 \mathrm{e}-4$ \\
\hline
\end{tabular}

which is two. On the other hand, the dynamic range of the power level is larger when $m$ is set at 1.5. So, there is a tradeoff between the convergence speed and the quality of the solution.

\section{CONCLUSION}

In this paper, a distributed power-control algorithm is proposed based on the concept of using a small amount of control information among the base stations. This algorithm does not require a common normalizing factor that needs to be communicated globally, and it exhibits desirable, monotonic convergence properties. A numerical study of the proposed algorithm was performed, and it shows that the speed of convergence can surpass a well-known distributed algorithm. Moreover, the proposed algorithm typically requires smaller power adjustments to achieve the balanced state. 


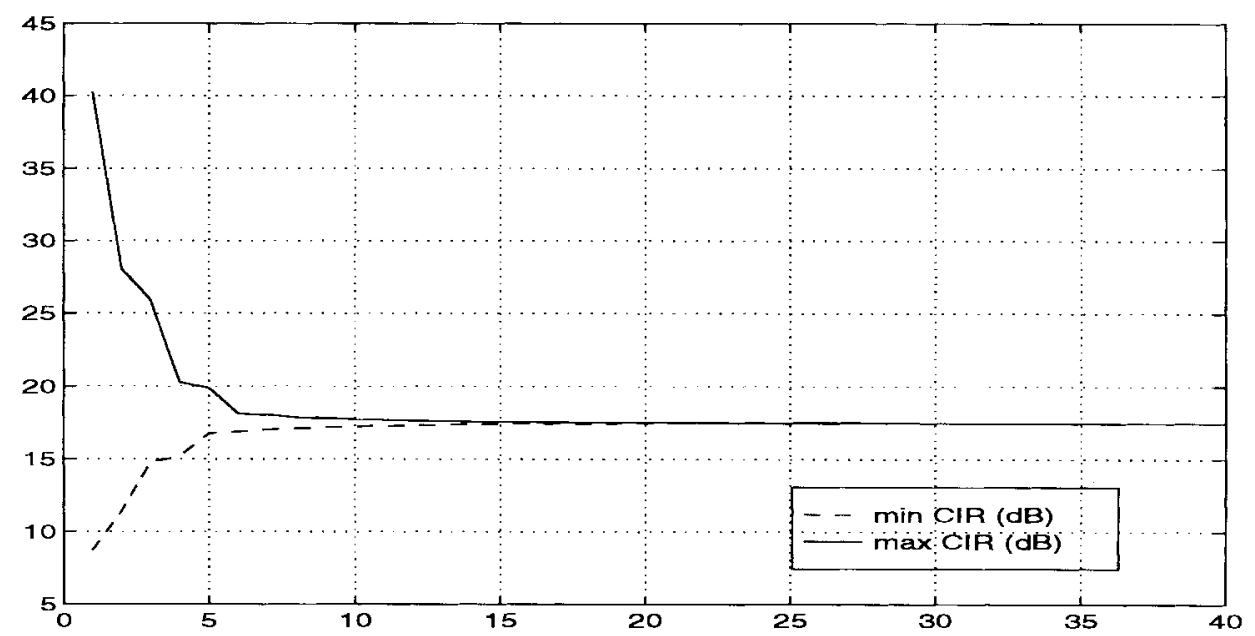

Fig. 5. Rate of convergence of the algorithm in [4]. Minimum and maximum C/I ratios are displayed.

\section{APPENDIX}

Proposition 4: For a two-cell system with quality threshold $\gamma_{0}$ set to zero, if an algorithm of the form

$$
\begin{aligned}
& P_{1}^{(n+1)}=f\left(\frac{\min \left(\Gamma_{1}^{(n)}, \Gamma_{2}^{(n)}\right)}{\Gamma_{1}^{(n)}}\right) P_{1}^{(n)} \\
& P_{2}^{(n+1)}=f\left(\frac{\min \left(\Gamma_{1}^{(n)}, \Gamma_{2}^{(n)}\right)}{\Gamma_{2}^{(n)}}\right) P_{2}^{(n)}
\end{aligned}
$$

converges in one step, then $f$ must be of the form

$$
f(x)=c \sqrt{x}
$$

where $c$ is an arbitrary constant.

Proof: Assume without loss of generality that $\Gamma_{1}^{(0)} \geq \Gamma_{2}^{(0)}$. Define

$$
x_{n}=\frac{\Gamma_{2}^{(n)}}{\Gamma_{1}^{(n)}} .
$$

It then follows from the definition that

$$
x_{n}=\frac{Z_{12}}{Z_{21}}\left(\frac{P_{2}^{(n)}}{P_{1}^{(n)}}\right)^{2} .
$$

If the algorithm converges in one step to the balanced solution, then, $x_{1}=1$. Hence,

$$
1=\frac{Z_{12}}{Z_{21}}\left(\frac{f(1) P_{2}^{(0)}}{f\left(x_{0}\right) P_{1}^{(0)}}\right)^{2}=x_{0}\left(\frac{f(1)}{f\left(x_{0}\right)}\right)^{2} .
$$

It follows that

$$
f(x)=f(1) \sqrt{x} .
$$

Proposition 5: For a two-cell system with quality threshold $\gamma_{0}$ set to zero, if an algorithm of the form

$$
\begin{aligned}
& P_{1}^{(n+1)}=f\left(\frac{\Gamma_{2}^{(n)}}{\Gamma_{1}^{(n)}}\right) P_{1}^{(n)} \\
& P_{2}^{(n+1)}=f\left(\frac{\Gamma_{1}^{(n)}}{\Gamma_{2}^{(n)}}\right) P_{2}^{(n)}
\end{aligned}
$$

converges in one step, then $f$ must be of the form

$$
f(x)=\sqrt[4]{x} h(\log x)
$$

where $h(x)$ is an even function, that is, $h(x)=h(-x)$.

Proof: By using arguments as in the proof of the previous proposition, one can show that

$$
1=\frac{Z_{12}}{Z_{21}}\left(\frac{f\left(1 / x_{0}\right) P_{2}^{(n)}}{f\left(x_{0}\right) P_{1}^{(n)}}\right)^{2}=x_{0}\left(\frac{f\left(1 / x_{0}\right)}{f\left(x_{0}\right)}\right)^{2} .
$$

Since the value $x_{0}$ can be any positive real number, $f$ must satisfy the equation

$$
f(x)=\sqrt{x} f(1 / x)
$$

for any positive real $x$.

To solve (34), define $g(s)=f\left(e^{s}\right)$. Notice that $g$ can take any real number as an argument. Equation (34) then becomes

$$
g(s)=e^{s / 2} g(-s)
$$

for any real $s$.

Define $h(s)=e^{-s / 4} g(s)$. Then, (35) becomes

$$
h(s)=h(-s)
$$

that is satisfied if and only if $h$ is an even function. Since $f(x)=$ $\sqrt[4]{x} h(\log x)$, the proposition then follows.

\section{REFERENCES}

[1] J. M. Aein, "Power balancing in systems employing frequency reuse," COMSAT Tech. Rev., vol. 3, no. 2, pp. 277-299, 1973.

[2] R. W. Nettleton and H. Alavi, "Power control for spread-spectrum cellular mobile radio system," in Proc. IEEE Veh. Technol. Conf., 1983, pp. 242-246.

[3] J. Zander, "Distributed cochannel interference control in cellular radio systems," IEEE Trans. Veh. Technol., vol. 41, no. 3, pp. 305-311, 1992.

[4] S. A. Grandhi, R. Vijayan, and D. J. Goodman, "Distributed power control in cellular radio systems," IEEE Trans. Commun., vol. 42, nos. 2/3/4, pp. 226-228, 1994

[5] G. J. Foschini, "A simple distributed autonomous power control algorithm and its convergence," IEEE Trans. Veh. Technol., vol. 42, no. 4, pp. 641-646, 1993.

[6] F. R. Gantmacher, The Theory of Matrices, vol. 2. New York: Chelsea, 1959.

[7] D. Mitra, "An asynchronous distributed algorithm for power control in cellular radio systems," in Proc. 4th WINLAB Workshop 3rd Generation Wireless Information Networks, 1993. 